Gut and Liver, Vol. 9, No. 3, May 2015, pp. 370-380

\title{
Expression of TIM-3, Human $\beta$-defensin-2, and FOXP3 and Correlation with Disease Activity in Pediatric Crohn's Disease with Infliximab Therapy
}

\author{
Mi Jin Kim*, Woo Yong Lee ${ }^{\dagger}$, and Yon Ho Choe \\ ${ }^{*}$ Department of Pediatrics, Ilsan Paik Hospital, Inje University College of Medicine, Goyang, Departments of ${ }^{\dagger}$ Surgery and ${ }^{\ddagger}$ Pediatrics, Samsung \\ Medical Center, Sungkyunkwan University School of Medicine, Seoul, Korea
}

See editorial on page 263.

Background/Aims: This study investigated the expression of $\mathrm{T}$ cell immunoglobulin- and mucin-domain-containing molecule 3 (TIM-3), human $\beta$-defensin (HBD)-2, forkhead box protein 3 (FOXP3), and the frequency of $\mathrm{CD} 4^{+} \mathrm{CD} 25^{+} \mathrm{FOXP3}^{+}$ regulatory $T$ cells (Tregs) in children with Crohn's disease (CD) during infliximab therapy. Methods: We enrolled $20 \mathrm{CD}$ patients who received infliximab treatment for 1 year. Peripheral blood and colonic mucosal specimens were collected from all $C D$ patients and from healthy control individuals. Results: A significant difference in TIM-3 mRNA expression was evident in peripheral blood mononuclear cells and colonic mucosa between $C D$ patients before infliximab therapy and the healthy controls ( $p<0.001$ and $p=0.005$, respectively). A significant difference in HBD-2 mRNA expression was found in colonic mucosa between $C D$ patients before infliximab therapy and the healthy controls $(p=0.013)$. In the active phase of $\mathrm{CD}$, at baseline, the median percentage of $\mathrm{T}$ cells that were $\mathrm{CD}_{25} 5^{+} \mathrm{FOXP3}^{+}$was $1.5 \%$ (range, $0.32 \%$ to $3.49 \%$ ), which increased after inflixmab treatment for 1 year to $2.2 \%$ (range, $0.54 \%$ to $5.02 \%)(p=0.008)$. Conclusions: Our study suggests that both the adaptive and innate immune systems are closely linked to each other in CD pathogenesis. And the results of our study indicate that it could be a useful therapeutic tool, where restoration of TIM-3, HBD-2 and the function of Tregs may repair the dysfunctional immunoregulation in CD. (Gut Liver 2015;9:370-380)

Key Words: Crohn disease; Infliximab; T-cell immunoglobulinand mucin-domain-containing molecule 3; forkhead box protein 3; Human beta-defensins-2

\section{INTRODUCTION}

Crohn's disease (CD) is a multifactorial disease of unknown etiology, which is characterized by chronic relapsing inflammation of the gastrointestinal tract. Immune, genetic and environmental factors are thought to contribute to CD. ${ }^{1}$ Disease symptoms caused by an 'over-reactive' immune response at the mucosa may be successfully dampened by corticosteroids, methotrexate, thiopurines or tumor necrosis factor (TNF) antibody. ${ }^{2}$ Therefore, immunoregulatory agents remain the mainstay of conservative therapy in $\mathrm{CD}$. However, the achievement of both induction and long-term maintenance of remission are still unsatisfactory. ${ }^{3}$

Subgroups of $\mathrm{T}$ helper cells are considered to play significant roles in the changed cytokine pattern and in mediation of chronic inflammation., ${ }^{4,5}$ TNF- $\alpha$ secreted from Th1 and Th17 cells plays a central role in the pathogenesis of mucosal inflammation in CD and seems to be at the apex of the inflammatory cascade. ${ }^{6}$ Generally, changed Th1 activation of cellular immunity is thought to play an important role in the pathogenesis of CD. ${ }^{7}$

T-cell immunoglobulin- and mucin-domain-containing molecule 3 (TIM-3) is a novel transmembrane protein that is involved in the regulation of Th1-cell-mediated immunity. ${ }^{8}$ In mice, TIM3 is a molecule expressed specifically on terminally differentiated Th1 cells, but not on Th2 cells, which negatively regulates Th1 responses. ${ }^{9,10}$ Furthermore, the newly identified Th17 subset expresses TIM-3, but at lower levels than Th1 cells. ${ }^{11,12}$ Galectin 9 (Gal-9), a $\beta$-galactoside-binding animal lectin belonging to the galectin family, is a physiological ligand of TIM-3 and induces apoptosis of TIM-3 expressing cells in vitro and in vivo. ${ }^{13}$ The negative regulatory effects of the TIM-3 pathway were recently investigated in a murine 2,4,6-trinitrobenzene sulfonic

Correspondence to: Yon Ho Choe

Department of Pediatrics, Samsung Medical Center, Sungkyunkwan University School of Medicine, 81 Irwon-ro, Gangnam-gu, Seoul 135-710, Korea

Tel: +82-2-3410-3539, Fax: +82-2-3410-0043, E-mail: i101016@skku.edu

Received on July 8, 2013. Revised on February 6, 2014. Accepted on February 24, 2014. Published online on July 25, 2014

pISSN 1976-2283 eISSN 2005-1212 http://dx.doi.org/10.5009/gnl13408

@ This is an Open Access article distributed under the terms of the Creative Commons Attribution Non-Commercial License (http://creativecommons.org/licenses/by-nc/3.0) which permits unrestricted non-commercial use, distribution, and reproduction in any medium, provided the original work is properly cited. 
acid-induced colitis model. ${ }^{14}$ However, the immunoregulatory effects of TIM-3 on patients with CD according to the treatment have not yet been investigated.

Modulation of regulatory $\mathrm{T}$ cells (Tregs) has considerable potential as an intervention for immune disorders. ${ }^{15}$ Naturally occurring $\mathrm{CD}^{+} \mathrm{CD}_{2} 5^{+}$Tregs are essential for the maintenance of mucosal tolerance. ${ }^{16}$ Colitis occurs in mice in which Tregs fail to develop properly, and transfer of Tregs can cure ongoing colitis. ${ }^{17-19} \mathrm{CD} 4^{+} \mathrm{CD} 25^{+}$Tregs express forkhead box protein 3 (FOXP3), a member of the fork-winged helix family of transcription factors, which is the major marker and functional regulator of natural Tregs development and function. ${ }^{19,20}$

In the intestinal lamina propria, Tregs may have evolved to suppress immune responses to resident commensal microbes. Therefore, lamina propria $\mathrm{CD} 4^{+}$and $\mathrm{CD} 25^{+}$Tregs might be involved in mucosal homeostasis. ${ }^{16}$ How the mucosal homeostasis is disrupted in $\mathrm{CD}$ to drive the development of the disease is unknown.

Human $\beta$-defensins (HBDs) are a group of evolutionarily conserved antimicrobial peptides that play a major role in innate host defense at various mucosal surfaces including the gastrointestinal tract. ${ }^{21}$ In the colon, epithelial cells and plasma cells in the lamina propria express HBDs. Epithelial cells constitutively express HBD-1 while expressions of HBD-2, -3 , and -4 are induced by various inflammatory and bacterial stimuli. ${ }^{22,23}$ Colonic plasma cells also express HBD-2, -3 , and -4 . However, it is unclear whether this expression is constitutive or inducible. Therefore, it is not surprising that impaired expression and/ or function of HBD has been implicated in the development of several diseases, including $\mathrm{CD}$.

The innate and adaptive immune system play a key role in the pathogenesis of $\mathrm{CD}^{24}$ However, cross-regulation of the innate and adaptive immune system has been widely-overlooked in pathogenesis research. In the intestine, innate immunity includes the epithelial barrier and phagocytic cells within the lamina propria (e.g., defensin, macrophages, and dendritic cells). ${ }^{25}$
T lymphocytes represent the key cell population of the adaptive immunity arm. $\mathrm{T}$ cells become activated, secrete cytokines and affect all other cell types within a local environment. ${ }^{26,27}$

The aim of this study was to investigate the expression of HBD-2, which is representative of innate immunity in CD, and TIM-3 and FOXP3, which are associated with adaptive immunity, and the frequency of $\mathrm{CD} 4^{+} \mathrm{CD} 25^{+} \mathrm{FOXP}^{+}$Tregs in the colonic mucosa and/or the peripheral blood of children with $\mathrm{CD}$ during infliximab therapy. Ultimately, we attempted to demonstrate the recovery and cross-regulation of innate and adaptive immunity during infliximab therapy in pediatric CD patients.

\section{MATERIALS AND METHODS}

\section{Subjects}

Among pediatric patients who were diagnosed with CD in accordance with the European Society for Pediatric Gastroenterology, Hepatology and Nutrition-Porto criteria ${ }^{28}$ at the Samsung Medical Center between January 2009 and January 2011, we enrolled 20 patients who received infliximab treatment for 1 year and who achieved clinical remission during the treatment period. Disease activity was graded according to the Pediatric Crohn's Disease Activity Index (PCDAI) (Fig. 1). ${ }^{29}$ As a healthy control group, 20 children with macroscopically and histologically normal mucosa as well as no evidence of any underlying gastrointestinal condition were recruited.

The monoclonal immunoglobulin G1 chimeric antibody directed against TNF- $\alpha$ (infliximab; Schering-Plough, Kenilworth, NJ, USA) was administered by intravenous infusion at a dose of $5 \mathrm{mg} / \mathrm{kg}$ at weeks 0,2 , and 6 , and this course was repeated every 8 weeks for 10 months thereafter.

This study was approved by the Institutional Review Board of the Samsung Medical Center. All participants provided written informed consent before participation in this study.

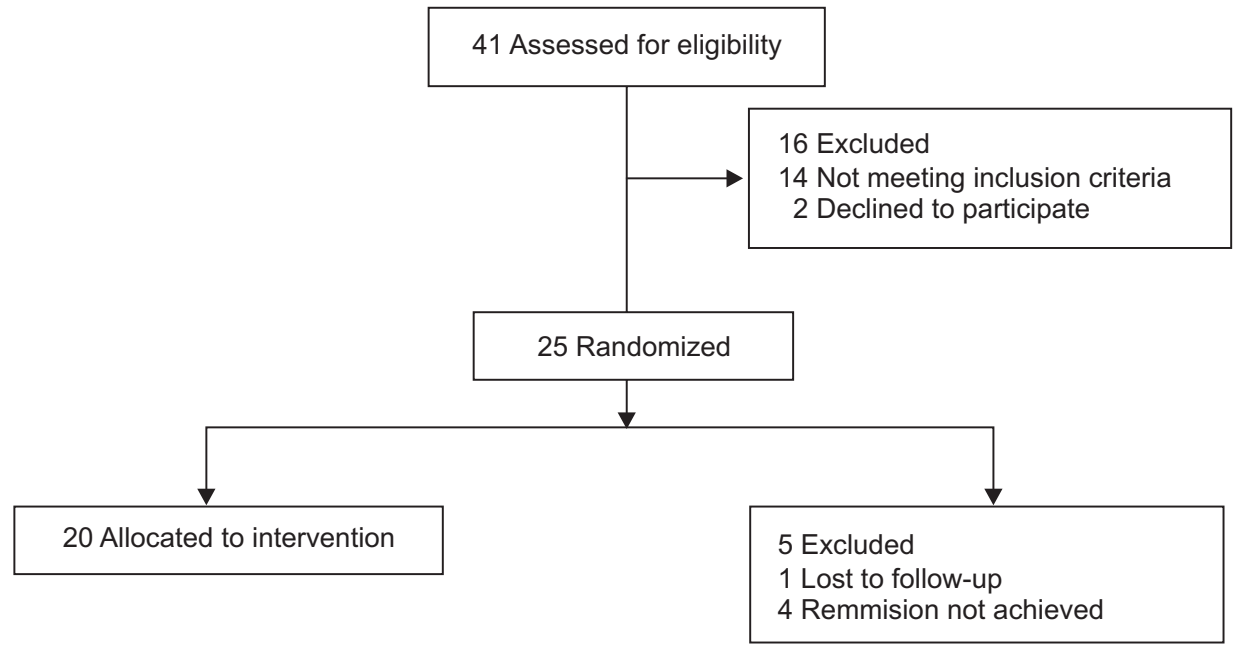

Fig. 1. Diagrammatic flow of the study. 


\section{Peripheral blood collection}

Peripheral blood for TIM-3 mRNA expression, Tregs assessment and cytokine detection by enzyme-linked immunosorbent assay (ELISA) was collected from all CD patients and healthy control individuals. In patients with $\mathrm{CD}$, blood collection was performed before infliximab therapy and after the eight course of infliximab therapy.

\section{Colonic mucosal biopsy}

Colonic mucosal specimens from all CD patients and healthy control individuals were assessed for mRNA expression of TIM3, HBD-2, and FOXP3, and for immunohistochemical analysis. Colonoscopy was performed by single dedicated pediatric gastroenterologist, who collected one or two additional biopsies within close proximity of the area in which biopsies were taken for routine histology. In patients with $\mathrm{CD}$, mucosal biopsies were performed before infliximab therapy and after the eighth course of infliximab therapy. The specimens were frozen and stored at $-80^{\circ} \mathrm{C}$ for RNA isolation.

\section{Flow cytometry analysis}

Peripheral blood mononuclear cells (PBMCs) were freshly isolated from whole blood by Ficoll-Plaque (GE Healthcare, Uppsala, Sweden) density gradient centrifugation. For immunostaining, phycoerythrin (PE)-Cy5-conjugated antibody against CD4 (mouse IgG1, clone RPA-T4; BD Pharminogen, Franklin Lakes, NJ, USA), PE-conjugated antibody against CD25 (mouse IgG1, clone M-A251; BD Pharminogen) and allophycocyanin (APC)conjugated antibody against FOXP (rat IgG2a, clone PCH101; eBioscience, San Diego, CA, USA) were employed. All antibodies were titrated against recommended isotype controls. The isolated mononuclear cells were washed and initially stained for surface CD4 and CD25, with optimal amounts of CD4-PE and $\mathrm{CD} 25-\mathrm{PE}$, for 30 minutes at $4^{\circ} \mathrm{C}$. The cells were subsequently stained for intracellular FOXP3 using FOXP3- APC, according to the manufacturer's instructions for fixation, permeabilization, and staining. Cells were then washed once in phosphate-buffered saline and expression of cell-surface or intracellular markers was assessed using a FACSCalibur flow cytometer (Becton Dickinson, San Diego, CA, USA) data were analyzed using Cell Quest software (Becton Dickinson).

\section{Reverse transcription-polymerase chain reaction}

Total RNA for FOXP3 from the frozen colonic tissue was isolated using Trizol reagent (Invitrogen, Carlsbad, CA, USA). RNA was extracted using an RNeasy Mini Kit (Qiagen, Valencia, CA, USA) according to the manufacturer's instructions. The amount and purity of the obtained RNA was determined by measurement of optical density at 260 and $280 \mathrm{~nm}$. The samples were treated with RNase-free DNase I (Qiagen) before reverse transcription to eliminate contaminating genomic DNA.
Reverse transcription-polymerase chain reaction (RT-PCR) was performed in a two-step procedure. The cDNA synthesis was carried out with $10 \mu \mathrm{L}$ total RNA, using reverse transcriptase and oligo/dT (SuperScript III; Invitrogen). The second step PCR was performed in a $50-\mu \mathrm{L}$ reaction volume containing $2-\mu \mathrm{L}$ cDNA, DNase/RNase-free water, 2x PCR buffer, $3 \mathrm{mM} \mathrm{MgCl}_{2}, 2$ $\mathrm{mM}$ deoxynucleotide triphosphate (dNTP), immolase DNA polymerase and $1 \mu \mathrm{M}$ of each primer. Primer sequences for FOXP3 were FOXP3 forward: 5'-CAG GAG CCC TTG TCG GAT-3', and FOXP3 reverse: 5'-CAG GAG CCC TTG TCG GAT-3'. After 2.5 minutes at $92^{\circ} \mathrm{C}, 35$ cycles of amplification were followed by 5 minutes extension at $72^{\circ} \mathrm{C}$. Each cycle consisted of denaturation at $92^{\circ} \mathrm{C}$ for 30 seconds, annealing at $56^{\circ} \mathrm{C}$ for 30 seconds and extension at $72^{\circ} \mathrm{C}$ for 30 seconds. PCR products were electrophoresed on $1 \%$ agarose gel stained with ethidium bromide and visualized by ultraviolet illumination analyzer. As a control, mRNA content for the housekeeping gene glyceraldehyde-3phophate dehydrogenase (GADPH) was analyzed using GAPDH forward: 5'-AGC CAC ATC GCT CAG ACA C-3' and GAPDH reverse: 5'-GAG GCA TTG CTG ATG ATC TTC-3' primers.

\section{Relative quantification of mRNA by real-time quantitative RT-PCR}

Total RNA for TIM-3 and HBD-2 from the frozen colonic tissue and/or peripheral blood was obtained using Trizol reagent (Invitrogen) according to the manufacturer's instructions. The amount and purity of the obtained RNA was determined using a ND-1000 spectrophotometer (Nanodrop Technologies, Wilmington, DE, USA). Reverse transcription was performed using SuperScript III First-Strand Synthesis System for RTPCR (Invitrogen). cDNA was prepared from $1 \mu \mathrm{g}$ of mRNA with oligo/dT according to the manufacturer's instructions. Real-time PCR was constructed using commercial available assays for the TIM-3 gene (Assay ID Hs00262170_m1, Gene bank accession number NM_032782.3; Applied Biosystems, Foster City, CA, USA), HBD-2 (Assay ID Hs00175474_m1, Gene bank accession number NM_004942.2), and human endogenous control GAPDH (Assay ID Hs99999905_m1, Gene bank accession number NM_002046.3) in combination with the TaqMan ${ }^{\circledR}$ Universal PCR Master Mix (Applied Biosystems), then PCR was performed in a 7900HT real-time PCR system (Applied Biosystems). Comparative analyses of each gene were performed using computer programs SDS 2.3 and RQ 2.1 (Applied Biosystems). The relative gene expression $(\mathrm{RQ})$ was calculated using the $2 \_\Delta \Delta \mathrm{CT}$ method.

\section{Cytokine detection assay by ELISA}

The TNF- $\alpha$ level was determined in the serum of both CD patients and healthy controls using a commercially available LINCOplex kit (Millipore, Billerica, MA, USA) in a Luminex analyzer, according to the manufacturer's instructions. The result was calculated through the Bio-Plex Manager Software (Bio-Rad Laboratories, Hercules, CA, USA) and the cytokine concentra- 
tion in plasma was expressed as $\mathrm{pg} / \mathrm{mL}$.

\section{Immunohistochemical analysis}

Four micrometer-thick paraffin sections of each intestinal mucosa sample from the colon of $\mathrm{CD}$ patients and controls were cut. Section were deparaffinized, blocked for peroxidase activity with $0.3 \% \mathrm{H}_{2} \mathrm{O}_{2}$ and rinsed with dextrose water. Antigen retrieval was performed by microwave pressure cooking for 3 minutes in Tris-buffered saline (TBS, pH 7.4). For immunohistochemistry of FOXP3, sections were blocked for nonspecific binding, washed with wash buffer and incubated for 1 hour with 236A/ E7 FOXP3 monoclonal antibody (Abcam, Cambridge, UK), followed by streptavidin (SA)-horseradish peroxidase (HRP)-conjugated mouse immunoglobulin (Ig) using the Envision Polymer Mouse kit (DakoCytomation, Glostrup, Denmark) and diaminobenzidine (DAB) substrate (DakoCytomation) for detection of antibody binding. For immunohistochemistry of TIM-3, sections were blocked for nonspecific binding, washed with wash buffer and incubated for one hour with the biotinylated TIM3 antibody (R\&D Systems, Minneapolis, MN, USA), followed by incubation with the SA-HRP Ig using the ChemMate LSAB kit (DakoCytomation) and DAB substrate (DakoCytomation) for detection of antibody binding. Each slide was counterstained with Mayer's hematoxylin (Sigma-Aldrich, St. Louis, MO, USA) and mounted.

\section{Statistical analyses}

Statistical analyses were performed using Mann-Whitney U-test for unpaired samples and Wilcoxon signed-rank test for paired samples using SPSS 16.0 software (SPSS Inc., Chicago, IL, USA). Correlations were determined by the simple linear regression. A p-value of $<0.05$ was considered statistically significant.

Table 1. Subject Characteristics

\begin{tabular}{lccc}
\hline \multirow{2}{*}{ Characteristic } & Controls & \multicolumn{2}{c}{ Patients } \\
\cline { 3 - 4 } & & Before IFX Tx & After IFX Tx \\
\hline No. & 20 & 20 \\
Gender, male/female & $16 / 4$ & $16 / 4$ \\
Age, yr & $14.8 \pm 1.8$ & $14.9 \pm 1.9$ \\
Medical treatment & & 2 \\
$\quad$ Corticosteroids & \multicolumn{2}{c}{17} \\
5-ASA & \multicolumn{2}{c}{20} \\
Azathioprine & & $34.4 \pm 13.5$ & $0.8 \pm 1.2$ \\
PCDAI score & & $48.0 \pm 30.9$ & $11.7 \pm 11.6$ \\
ESR, mm/hr & $3.2 \pm 3.5$ & $0.1 \pm 0.2$ \\
CRP, mg/dL & & \multicolumn{2}{c}{} \\
\hline
\end{tabular}

Data are expressed in number or mean \pm SD.

IFX Tx, infliximab therapy; PCDAI, Pediatric Crohn's Disease Activity Index; ESR, erythrocyte sedimentation rate; CRP, C-reactive protein.

\section{RESULTS}

\section{Baseline characteristics and clinical outcomes}

The 40 children comprised $20 \mathrm{CD}$ patients (16 males, four females) and 20 healthy controls (16 males, four females). The mean age was $14.8 \pm 1.8$ years for the healthy controls and $14.9 \pm 1.9$ years for the CD patients; there were no statistical differences in mean ages between these groups (Table 1).

After infliximab treatment for 1 year, disease activity, according to PCDAI score, decreased from $34.4 \pm 13.5$ at the baseline to $0.75 \pm 1.2$ ( $\mathrm{p}<0.001$ ), furthermore, erythrocyte sedimentation rate declined from $48.0 \pm 30.9 \mathrm{~mm} / \mathrm{hr}$ to $11.7 \pm 11.6 \mathrm{~mm} / \mathrm{hr}(\mathrm{p}<0.001)$, and C-reactive protein declined from $3.2 \pm 3.5 \mathrm{mg} / \mathrm{dL}$ to $0.1 \pm 0.24$ $\mathrm{mg} / \mathrm{dL}(\mathrm{p}<0.001)$ (Table 1).

\section{Expression of cytokine in peripheral blood from CD pa- tients before and after infliximab therapy compared to normal controls and its correlation with disease activity}

TIM-3 is preferentially expressed on differentiated Th1 cells and regulates Th1 response negatively. ${ }^{9,10}$ Disruption of this negative feedback loop is postulated to induce an 'over-reactive' immune response. To test the hypothesis that TIM-3 dysregulation contributes to the pathogenesis of $\mathrm{CD}$, we first had to determine whether the Th1 immune response was augmented in CD. We performed an experiment to measure the level of TNF- $\alpha$ in the plasma of CD patients before and after infliximab therapy and healthy controls using cytokine-specific ELISAs. The plasma level of TNF- $\alpha$ in CD patients before infliximab therapy was higher than that of the healthy controls ( $p=0.026$ ) (Fig. 2).

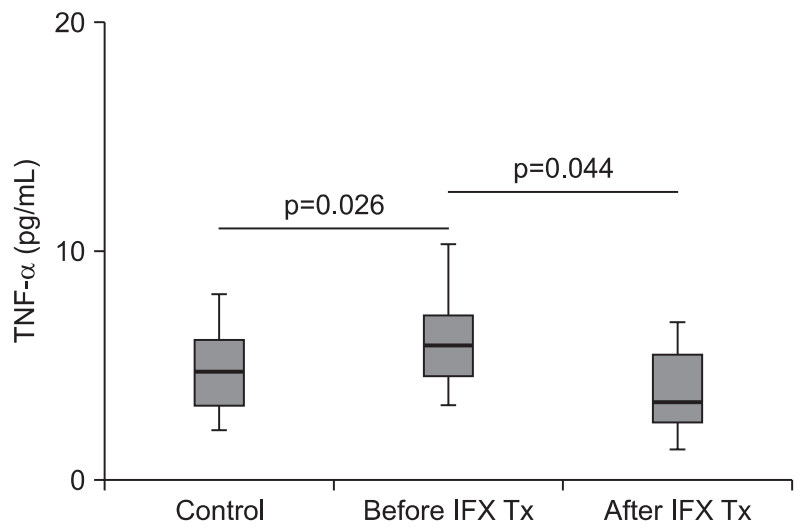

Fig. 2. Expression of tumor necrosis factor $\alpha(\mathrm{TNF}-\alpha)$ in peripheral blood and its correlation with disease activity. The plasma level of TNF- $\alpha$ in the Crohn's disease (CD) patients and healthy controls was determined by enzyme-linked immunosorbent assay. The TNF- $\alpha$ levels in the serum of CD patients before infliximab therapy (IFX $\mathrm{Tx}$ ) was higher than that of the healthy controls $(\mathrm{p}=0.026)$. After the IFX Tx, the level of TNF- $\alpha$ was decreased compared with that before therapy $(p=0.044)$. Box plots define the values for the median, range, and 25th and 75th percentiles. The range is represented by the upper and lower limits of the vertical lines. 
After infliximab therapy, the level of TNF- $\alpha$ in CD patients was decreased compared to that before therapy ( $p=0.044)$ (Fig. 2). A correlation was not found between TNF- $\alpha$ level in plasma from CD patients and disease activity as measured by PCDAI during infliximab therapy $\left(r^{2}=0.086, p=0.067\right)$.

\section{Expression of TIM-3 in PBMC from CD patients before and after infliximab therapy compared to normal con- trols and its correlation with disease activity}

TIM-3 mRNA expression in PBMC was examined using

A

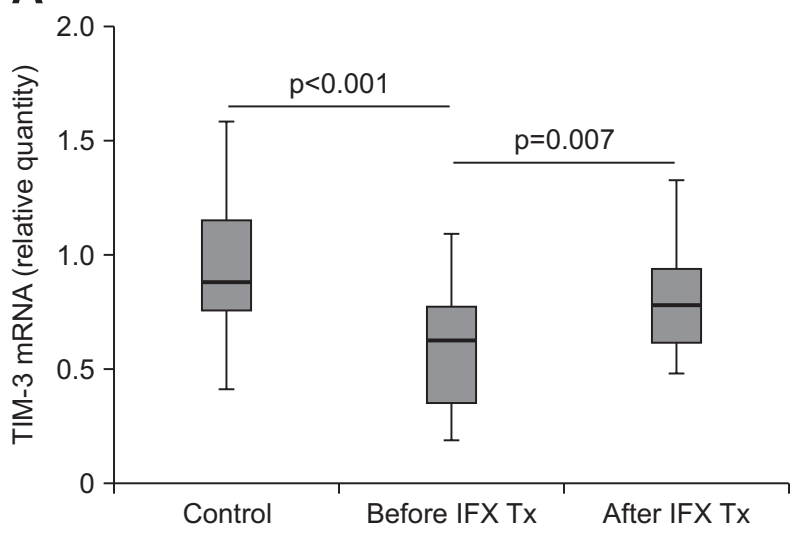

quantitative real-time RT-PCR. A significant difference in TIM3 mRNA expression was found between PBMC from CD patients before infliximab therapy and that from healthy controls $(\mathrm{p}<0.001)$ (Fig. 3A). After infliximab therapy, TIM-3 mRNA expression in $\mathrm{CD}$ patients was increased compared to that before therapy ( $\mathrm{p}=0.007$ ) (Fig. 3A). A negative correlation was found between TIM-3 mRNA expression in PBMC from CD patients and PCDAI during infliximab therapy $\left(\mathrm{r}^{2}=0.201, \mathrm{p}=0.004\right)$ (Fig. 3B).

B

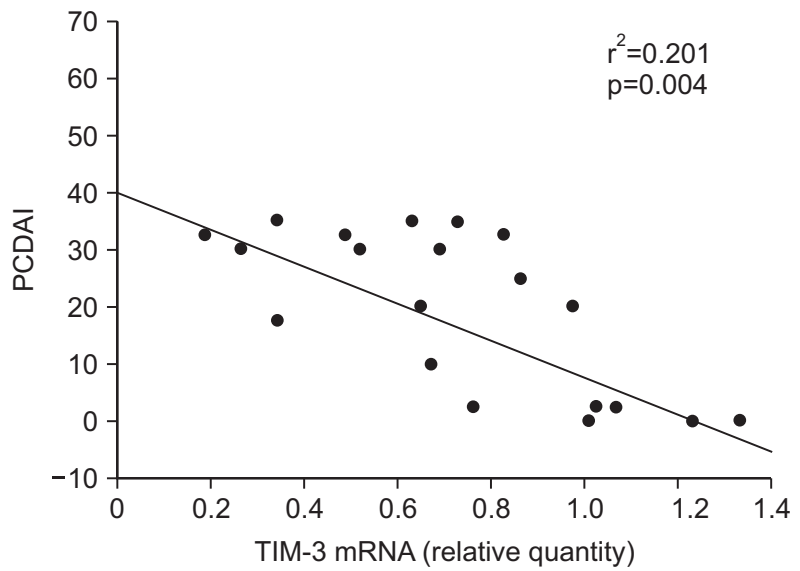

Fig. 3. Expression of $\mathrm{T}$ cell immunoglobulin- and mucin-domain-containing molecule 3 (TIM-3) mRNA in peripheral blood mononuclear cells (PBMCs) and its correlation with disease activity. (A) Expression of TIM-3 mRNA in the PBMCs of Crohn's disease (CD) patients and healthy controls was determined by real-time reverse transcription-polymerase chain reaction. The TIM- 3 mRNA expression in the PBMCs of CD patients before infliximab therapy (IFX Tx) was significantly lower than that in the healthy controls $(\mathrm{p}<0.001)$. After IFX Tx, the TIM- 3 mRNA expression was increased compared with that before therapy $(\mathrm{p}=0.007)$. Box plots define the values for the median, range, and 25th and 75th percentiles. The range is represented by the upper and lower limits of the vertical lines. (B) Negative correlation of the TIM- 3 mRNA expression in the PBMCs of CD patients with respect to the Pediatric Crohn's Disease Activity Index (PCDAI) during IFX Tx $\left(\mathrm{r}^{2}=0.201, \mathrm{p}=0.004\right)$.

A

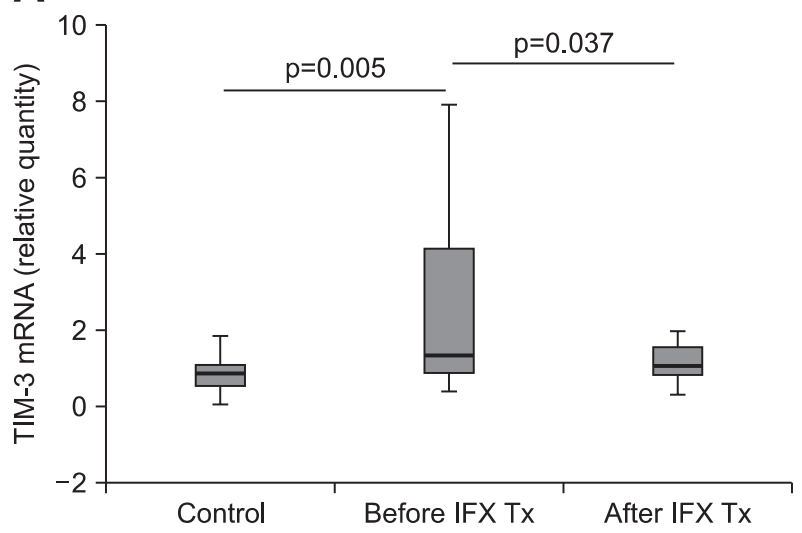

B

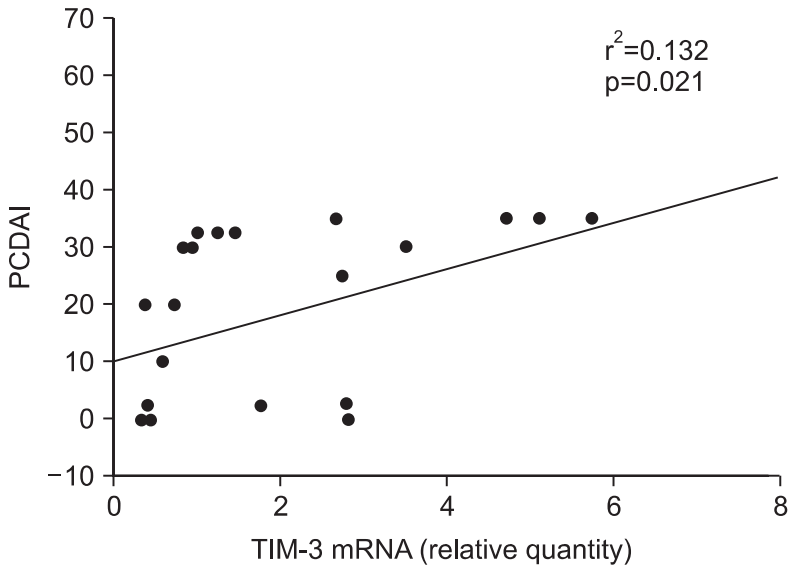

Fig. 4. Expression of $\mathrm{T}$ cell immunoglobulin- and mucin-domain-containing molecule 3 (TIM-3) mRNA in the colonic mucosa and its correlation with disease activity. (A) The expression of TIM-3 mRNA in the colonic mucosa of the Crohn's disease (CD) patients and healthy controls was determined by real-time reverse transcription-polymerase chain reaction. The TIM-3 mRNA expression in the colonic mucosa of CD patients before infliximab therapy (IFX Tx) was significantly higher than that in the healthy controls $(\mathrm{p}=0.005)$. After IFX Tx, TIM-3 mRNA expression was decreased compared with that before therapy $(\mathrm{p}=0.037)$. Box plots define the values for the median, range, and 25th and 75th percentiles. The range is represented by the upper and lower limits of the vertical lines. (B) Positive correlation of TIM-3 mRNA expression in the colonic mucosa of CD patients with respect to the Pediatric Crohn's Disease Activity Index (PCDAI) during IFX Tx $\left(r^{2}=0.132, p=0.021\right)$. 


\section{Expression of TIM-3 in the colonic mucosa from CD pa- tients before and after infliximab therapy compared to normal controls and its correlation with disease activity}

TIM-3 mRNA expression in the colonic mucosa was examined using quantitative real-time RT-PCR. A significant difference in TIM-3 mRNA expression was found between colonic mucosa from $\mathrm{CD}$ patients before infliximab therapy and that from healthy controls ( $\mathrm{p}=0.005)$ (Fig. 4A). After infliximab therapy, TIM-3 mRNA expression in CD patients was decreased compared to that before therapy ( $\mathrm{p}=0.037$ ) (Fig. 4A). A positive correlation was found between TIM-3 mRNA expression in the colonic mucosa from $\mathrm{CD}$ patients and PCDAI during infliximab therapy ( $\left.r^{2}=0.132, p=0.021\right)$ (Fig. $\left.4 B\right)$.

\section{Expression of HBD-2 in the colonic mucosa from CD pa- tients before and after infliximab therapy compared to normal controls and its correlation with disease activity}

HBD-2 mRNA expression in the colonic mucosa was examined using quantitative real-time RT-PCR. A significant difference in HBD-2 mRNA expression was found between colonic mucosa from $\mathrm{CD}$ patients before infliximab therapy and that
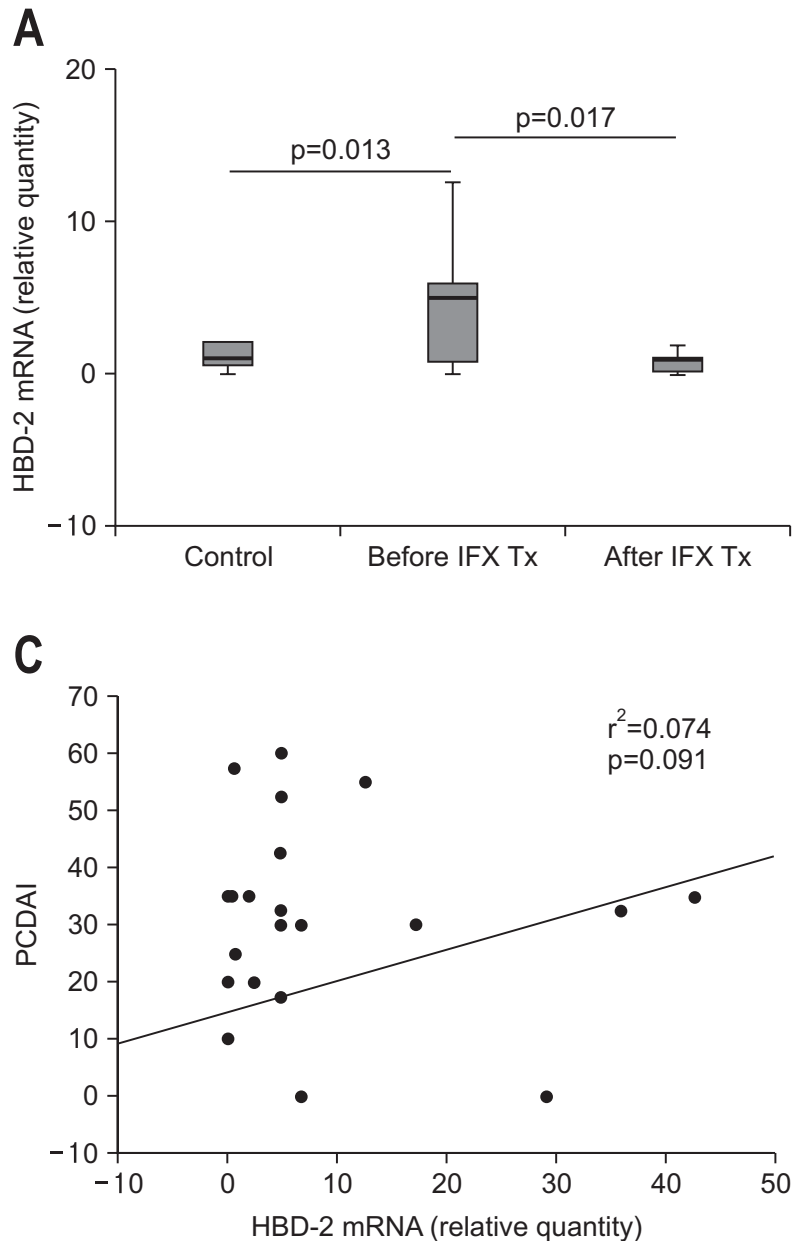

from healthy controls ( $\mathrm{p}=0.013$ ) (Fig. 5A). After infliximab therapy, HBD-2 mRNA expression in CD patients was decreased compared to that before therapy $(\mathrm{p}=0.017)$ (Fig. 5A). A negative correlation was found between TIM-3 mRNA expression in PBMC and HBD-2 mRNA expression in colonic mucosa from CD patients $\left(r^{2}=0.344, p=0.007\right)$ (Fig. 5B). However, a correlation was not found between HBD-2 mRNA expression in colonic mucosa from $\mathrm{CD}$ patients and PCDAI during infliximab therapy $\left(r^{2}=0.074, p=0.091\right)$ (Fig. 5C).

\section{Increments of $\mathrm{CD}^{2} 5^{+} \mathrm{FOXP3}^{+}$Tregs in PBMC after inflix- imab therapy and correlation with disease activity}

Representative FACS analysis of FOXP3 and CD25 expression, gated on the $\mathrm{CD}^{+} \mathrm{T}$ cells, by lymphocytes is shown in Fig. $6 \mathrm{~A}$. In the active phase of $\mathrm{CD}$, at baseline, the median percentage of $\mathrm{T}$ cells that were $\mathrm{CD} 25^{+} \mathrm{FOXP}^{+}$was $1.5 \%$ (range, $0.32 \%$ to $3.49 \%$ ), which increased after inflixmab treatment for 1 year up to $2.2 \%$ (range, $0.54 \%$ to $5.02 \%)(p=0.008)$ (Fig. 6B). The change in disease activity, measured as PCDAI during inflixmab therapy was inversely correlated with the upregulation of CD25 F0XP3 ${ }^{+}$Tregs $\left(r^{2}=0.604, p<0.001\right)$ (Fig. 6C).

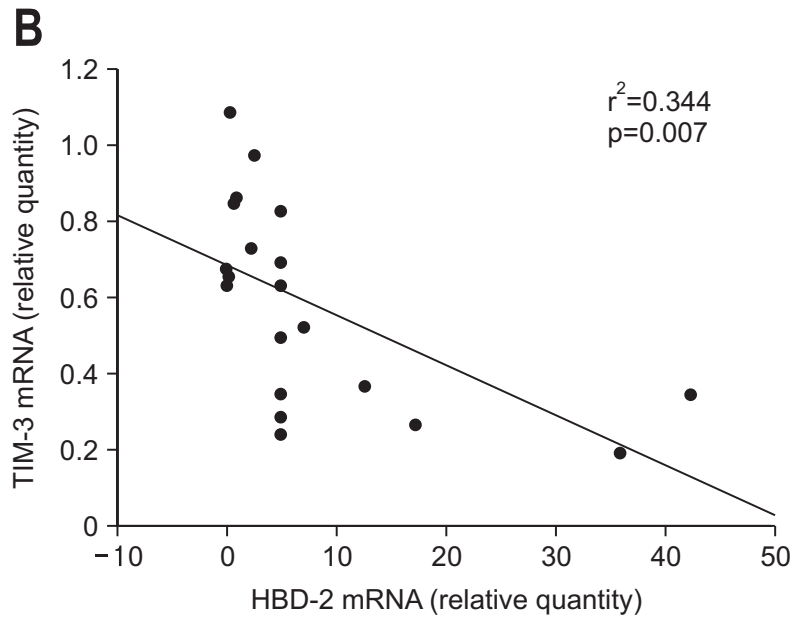

Fig. 5. Expression of human $\beta$-defensin (HBD)-2 mRNA in the colonic mucosa and its correlation with disease activity. (A) The expression of HBD-2 mRNA in the colonic mucosa of the Crohn's disease (CD) patients and healthy controls was determined by real-time reverse transcription-polymerase chain reaction. The HBD-2 mRNA expression in the colonic mucosa of $\mathrm{CD}$ patients before infliximab therapy (IFX Tx) was significantly higher than that in the healthy controls $(p=0.013)$. After IFX Tx, HBD-2 mRNA expression was decreased compared with that before therapy $(p=0.017)$. Box plots define the values for the median, range, and 25th and 75th percentiles. The range is represented by the upper and lower limits of the vertical lines. (B) A negative correlation was found between the T cell immunoglobulin- and mucin-domain-containing molecule 3 (TIM3) mRNA expression in peripheral blood mononuclear cells and the HBD-2 mRNA expression in the colonic mucosa in the CD patients $\left(r^{2}=0.344, p=0.007\right)$. (C) Correlation of HBD-2 mRNA expression in the colonic mucosa with the clinical disease activity index during IFX $\operatorname{Tx}\left(r^{2}=0.074, p=0.091\right)$. 


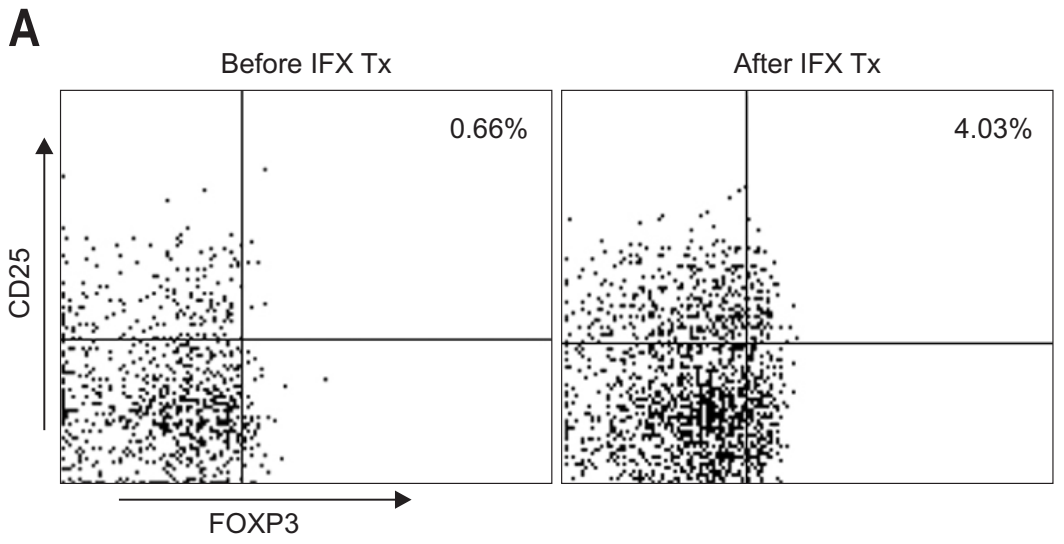

B

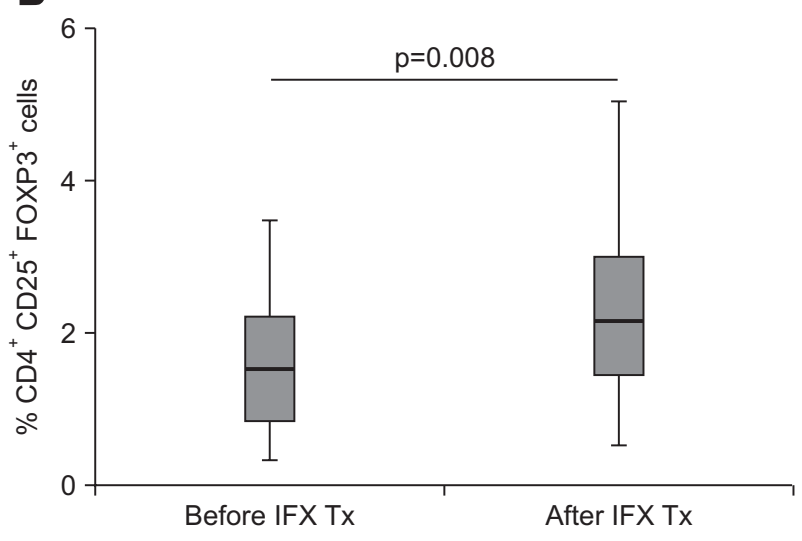

C

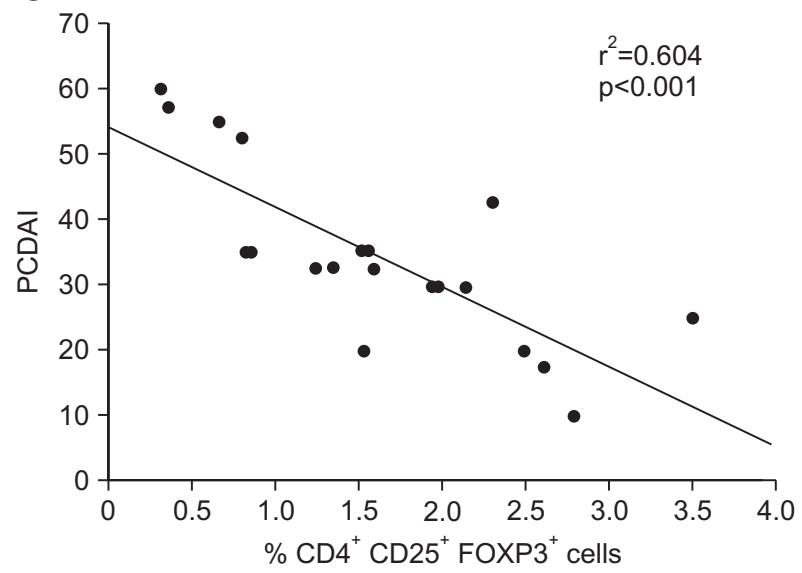

Fig. 6. $\mathrm{CD}^{+} \mathrm{CD}_{2} 5^{+} \mathrm{FOXP}^{+}$Tregs in peripheral blood. (A) Representative dot plot of forkhead box protein 3 (FOXP3) and CD25 staining in $\mathrm{CD} 4{ }^{+}$ peripheral blood lymphocytes from one patient before and after infliximab therapy (IFX Tx). (B) Comparison of the percentage of $\mathrm{CD}^{+} \mathrm{CD}^{2} 5^{+}$ FOXP3 $^{+}$cells before and after IFX Tx. Box plots define the values for the median, range, and 25th and 75th percentiles. The range is represented by the upper and lower limits of the vertical lines. (C) A nagative correlation of the percentage of $\mathrm{CD} 4^{+} \mathrm{CD} 25^{+} \mathrm{FOXP} 3^{+}$cells with respect to the Pediatric Crohn's Disease Activity Index (PCDAI) during IFX Tx $\left(\mathrm{r}^{2}=0.604, \mathrm{p}<0.001\right)$.

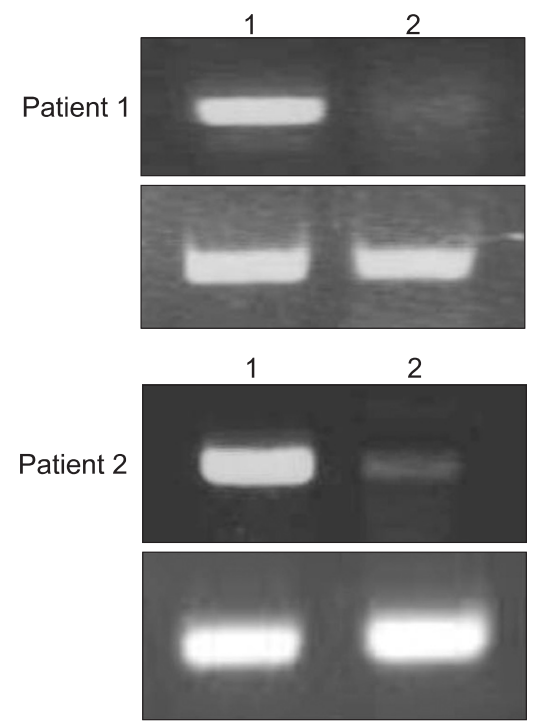

Fig. 7. Forkhead box protein 3 (FOXP3) mRNA expression in the colonic mucosa from two patients before and after infliximab therapy. The numerals 1 and 2 represent before and after infliximab therapy, respectively. The upper line is the FOXP3 mRNA band, and the lower line is the GAPDH band.

\section{Expression of FOXP3 in the colonic mucosa from CD pa- tients before and after infliximab therapy}

FOXP3 mRNA expression in the colonic mucosa was examined using RT-PCR. It was compared, in paired samples, before and after infliximab treatment. The FOXP3 mRNA band was more prominent before infliximab treatment than after treatment. This was evidence that FOXP3 mRNA expression in the colonic mucosa after infliximab therapy was decreased compared to that before therapy (Fig. 7).

\section{Immunohistochemical analysis of TIM-3 and FOXP3 in the colonic mucosa of CD patients before and after inf- liximab therapy compared to normal controls}

Colonic mucosa from CD patients before and after infliximab therapy and healthy controls were stained by immunohistochemical methods. The expression of TIM-3 and FOXP3 was detected histologically in the colonic mucosa of CD patients, and was decreased according to infliximab treatment. In contrast, no staining was observed in the colonic mucosa of healthy controls 

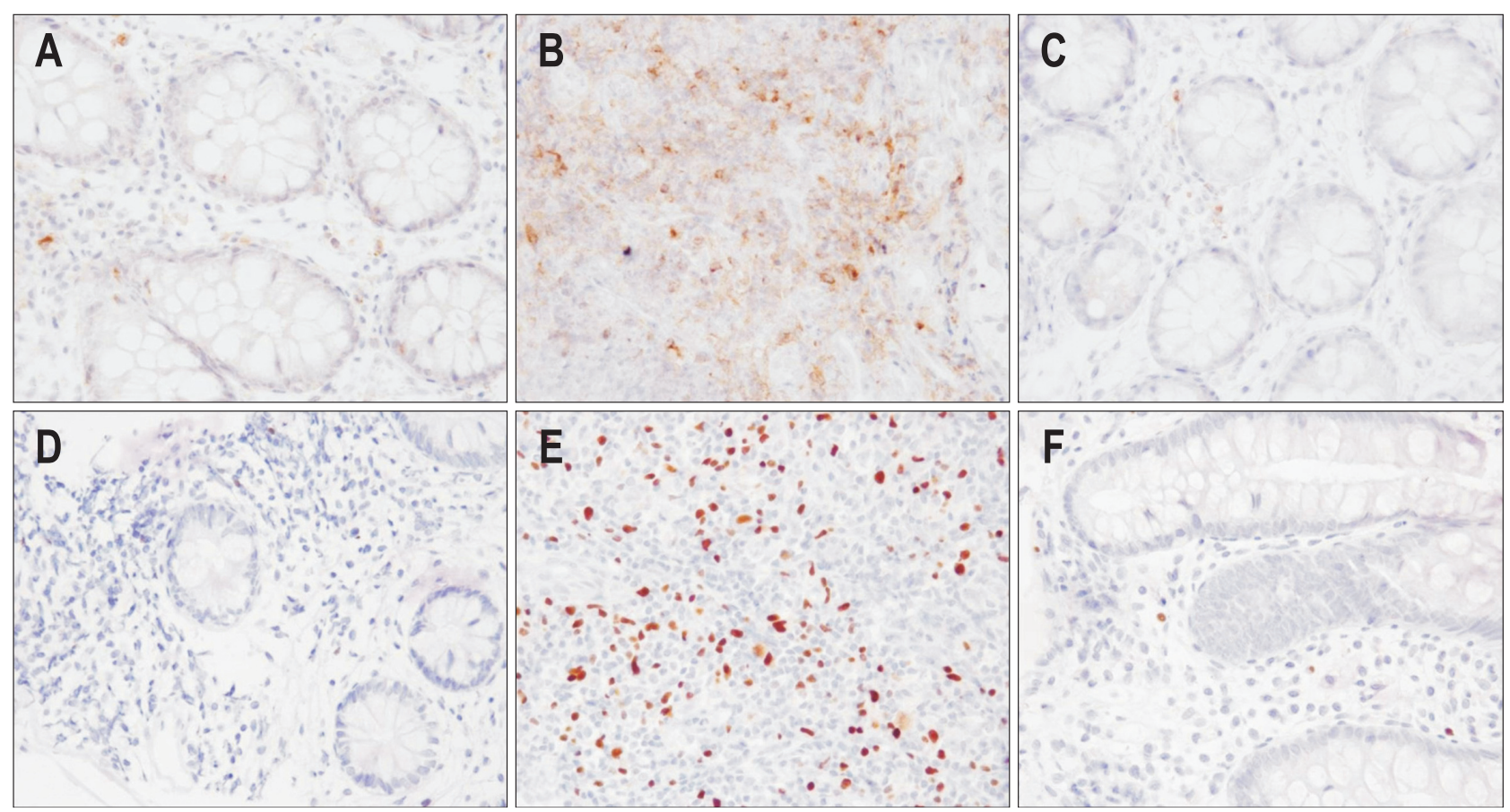

Fig. 8. Expression of the T cell immunoglobulin- and mucin-domain-containing molecule 3 (TIM-3) and forkhead box protein 3 (FOXP3) proteins in the colonic mucosa of patients with Crohn's disease (CD) before and after infliximab therapy and normal controls. The immunoreactive TIM-3 protein in normal controls (A) was compared with that in CD patients before (B) and after (C) infliximab therapy by immunohistochemical analysis using anti-TIM-3 antibodies as described in the Materials and Methods section. The immunoreactive FOXP3 protein in CD patients before (E) and after (F) infliximab therapy was compared with that in normal controls (D) by immunohistochemical analysis using anti-F0XP3 antibodies as described in the Materials and Methods section (×400).

(Fig. 8).

\section{DISCUSSION}

$\mathrm{CD}$ is the consequence of a dysregulated immune system. The immune system consists innate and adaptive immunity that have been studied separately for a long time. However, crossregulation of innate and adaptive immunity in $\mathrm{CD}$ has not been completely elucidated.

The role of the Th1 and Th2 polarization immune response in human disease has been unknown for a long time. ${ }^{30,31}$ Until very recently, Th1 and Th2 cells could only be identified functionally through the cytokines they secreted; there was no precise way to isolate viable Th1 or Th2 cells by virtue of a unique surface molecule. ${ }^{32}$ For this reason, Monney et al. ${ }^{8}$ generated and screened monoclonal antibodies for activity against Th1 and Th2 cells, and discovered a novel cell surface molecule, TIM-3, which contains an immunoglobulin- and a mucin-like domain and which is expressed on differentiated Th1 cells, but not on Th2 cells. Extending this observation, they further demonstrated that in vivo administration of antibody to TIM3 exacerbated the clinical and pathological severity of experimental autoimmune encephalomyelitis, a Th1/Th17 mediated autoimmune disease, and increased the number and activation level of macrophages. Their results indicate that TIM-3 may have an important role in the induction of autoimmune diseases by regulating macrophage activation and/or function. A shift toward Th1 activation of cellular immunity has been implicated in CD pathogenesis. ${ }^{7}$ TIM-3 is a key regulatory molecule for Th1 response and has been shown to inhibit Th1-mediated autoand alloimmune responses, and promote immunological tolerance. $^{9,10}$ Therefore, it is highly likely that TIM-3 plays a crucial role in the pathogenesis of $\mathrm{CD}$.

A previous study showed that low TIM-3 expression were found in PBMC from CD patients and healthy controls. However, TIM-3 expression on Th cells isolated from intestinal mucosa was significantly lower in CD patients than healthy controls. ${ }^{33}$ The present investigation revealed relatively higher TIM-3 mRNA expression from colonic mucosa in contrast to low expression on PBMC of CD patients before infliximab treatment, compared with healthy controls. We could not explain this discrepancy completely. In the present study, we performed quantitative real-time RT-PCR using whole colonic biopsy specimen instead of mononuclear cell isolation from colonic biopsy specimen. Therefore, further studies using mononuclear cell from colonic specimen are required in the near future. Another possible explanation in regard to our results is that low levels of TIM-3 on PBMC of CD patients before infliximab treatment may allow Th1 cells to escape Gal-9-induced cell death leading to chronic inflammation. Of note, our finding of a negative correlation be- 
tween TIM-3 mRNA expression from PBMC in CD patients with respect to PCDAI also corroborates this hypothesis. High levels of TIM-3 expression from colonic mucosa of CD patients before infliximab treatment may be explained by the location of active inflammation, and by the possibility of a differential expression of TIM-3 between the peripheral blood and the affected intestinal mucosa. After infliximab treatment for 1 year, TIM-3 mRNA expression decreased in colonic mucosa and increased in PBMC compared to that before treatment.

Although TIM-3 was first identified through a screen for Th1specific markers, recent findings have demonstrated that TIM3 may also be expressed on several other cell types, including cytotoxic CD8 ${ }^{+} \mathrm{T}$ cells, Th17, Tregs, monocytes, dendritic cells, microglia and mast cells. ${ }^{8,34-37}$ The exact mechanism by which TIM-3 influences T-cell tolerance is still unknown, but might involve the modulation of regulatory T-cell function. $\mathrm{CD}^{+}$ $\mathrm{CD} 25^{+}$Tregs are considered to play a crucial role as suppressors of immune-mediated reaction. The proinflammatory environment, which is rich with TNF- $\alpha$ and which is generated during the active status of $\mathrm{CD}$, can prevent viability or expansion of Tregs, reducing their frequency. ${ }^{38}$ This would result in a reduced regulatory activity of these cells and further amplification of the inappropriate immune response. In CD, exuberant TNF- $\alpha$ production may limit the activity of Tregs by binding to the TNF receptor-2 (TNFR2), and promote induction of immune reactivity and the effector phase of lymphocyte responses. Therefore, the loss of mucosal homeostasis with the increase of T-cell proliferation and the apoptosis of Tregs might be caused by the effect of TNF- $\alpha .^{39}$ The increased number of FOXP3 ${ }^{+}$Tregs after TNF- $\alpha$ neutralization with infliximab may be explained by a decrease in TNF- $\alpha$ that results in reduced activation of Tregs through TNFR2 and reduction of Tregs apoptosis. Presently, the frequency of $\mathrm{CD}^{+} \mathrm{CD}^{2} 5^{+} \mathrm{FOXP}^{+}$Tregs in PBMC increased after infliximab treatment and a negative correlation between the frequency of $\mathrm{CD}^{+} 25^{+} \mathrm{FOXP}^{+}$Tregs and PCDAI was evident.

To define the tissue specific effect of Tregs, we compared the FOXP3 mRNA expression, a master transcription factor for Tregs differentiation, in the colonic mucosa before and after infliximab therapy. In the mucosa, the expression of FOXP3 mRNA was increased during the active phase of $C D$ and decreased after infliximab treatment. These results suggest that, in $\mathrm{CD}$ patients, a proper tissue specific immune regulatory function is maintained by a local aggregation of Tregs. ${ }^{40-46}$ Presently, FOXP3 mRNA expression in the colonic mucosa was greater at the inflammatory site before infliximab treatment compared to the healed site during clinical remission after infliximab treatment. It is reasonable to assume that there is a positive association between TIM-3 and FOXP3 because of a similar pattern of alteration in PBMC and colonic tissue.

One of the most commonly-accepted hypotheses on CD etiology is that abnormalities in the innate immune response by the mucosa cause a loss of tolerance to commensal microbiota and alterations in the composition of the gut microbiota. As a consequence for this defect, the host immune system is overwhelmed by bacterial antigens, which lead to chronic immune-mediated intestinal injury. HBD have been shown to play a key role in intestinal innate host defence. ${ }^{47}$ Importantly, in addition to the well-documented antimicrobial properties of HBD, evidence is rapidly increasing on their role as potent immune modulators capable of enhancing inflammatory processes, hence potentially contributing to the onset and/or persistence of chronic intestinal inflammation. ${ }^{48}$ There is evidence that expression and regulation of HBD produced in the intestinal mucosa are altered in $\mathrm{CD} .^{21}$

The colonic epithelial expression of HBD-2 is induced in the presence of inflammatory stimuli. There is little HBD-2 expression by the epithelium of normal colon, but significantly increased expression in inflamed colon. ${ }^{49}$ Our results also show that HBD-2 mRNA expression of colonic mucosa in CD patients was higher than that of healthy controls and, after infliximab therapy, HBD-2 expression was decreased compared to that before therapy because of decreasing inflammatory stimuli. The present study revealed a negative correlation between TIM-3 mRNA expression in PBMC and HBD-2 mRNA expression in colonic mucosa from $\mathrm{CD}$ patients.

This study is the first investigation that has attempted to determine the clinical correlation with TIM-3, FOXP3, and HBD-2 expression, and cross-regulation of innate and adaptive immunity according to infliximab therapy. Collectively, our data suggest that both the adaptive and innate immune systems are closely linked to each other in CD pathogenesis. Further studies on larger groups of patients with $\mathrm{CD}$ receiving infliximab therapy are required to confirm these data, elucidate the mechanisms that underlie these observations and relate the clinical and immunological responses. Our study indicated that it could be a useful therapeutic tool, where restoration of TIM-3, HBD-2 and the function of Tregs may repair the dysfunctional immunoregulation in $\mathrm{CD}$.

\section{CONFLICTS OF INTEREST}

No potential conflict of interest relevant to this article was reported.

\section{ACKNOWLEDGEMENTS}

This study was supported by the Korea Healthcare Technology R\&D Project, Ministry for Health and Welfare Affairs, Republic of Korea (A092255), and Samsung Biomedical Research Institute (grant number: SBRI C-A6-229-3).

\section{REFERENCES}

1. Xavier RJ, Podolsky DK. Unravelling the pathogenesis of inflammatory bowel disease. Nature 2007;448:427-434. 
2. Travis SP, Stange EF, Lémann M, et al. European evidence based consensus on the diagnosis and management of Crohn's disease: current management. Gut 2006;55 Suppl 1:i16-i35.

3. Lémann M, Mary JY, Duclos B, et al. Infliximab plus azathioprine for steroid-dependent Crohn's disease patients: a randomized placebo-controlled trial. Gastroenterology 2006;130:1054-1061.

4. Mayer L. Evolving paradigms in the pathogenesis of IBD. J Gastroenterol 2010;45:9-16.

5. Mizoguchi A, Mizoguchi E. Inflammatory bowel disease, past, present and future: lessons from animal models. J Gastroenterol 2008;43:1-17.

6. Papadakis KA, Targan SR. Role of cytokines in the pathogenesis of inflammatory bowel disease. Annu Rev Med 2000;51:289-298.

7. Fiocchi C. Inflammatory bowel disease: etiology and pathogenesis. Gastroenterology 1998;115:182-205.

8. Monney L, Sabatos CA, Gaglia JL, et al. Th1-specific cell surface protein Tim-3 regulates macrophage activation and severity of an autoimmune disease. Nature 2002;415:536-541.

9. Anderson AC, Anderson DE. TIM-3 in autoimmunity. Curr Opin Immunol 2006;18:665-669.

10. Sánchez-Fueyo A, Tian J, Picarella D, et al. Tim-3 inhibits T helper type 1-mediated auto- and alloimmune responses and promotes immunological tolerance. Nat Immunol 2003;4:1093-1101.

11. Chen Y, Langrish CL, McKenzie B, et al. Anti-IL-23 therapy inhibits multiple inflammatory pathways and ameliorates autoimmune encephalomyelitis. J Clin Invest 2006;116:1317-1326.

12. Nakae S, Iwakura Y, Suto H, Galli SJ. Phenotypic differences between Th1 and Th17 cells and negative regulation of Th1 cell differentiation by IL-17. J Leukoc Biol 2007;81:1258-1268.

13. Zhu C, Anderson AC, Schubart A, et al. The Tim-3 ligand galectin-9 negatively regulates $\mathrm{T}$ helper type 1 immunity. Nat Immunol 2005;6:1245-1252.

14. Li X, Chen G, Li Y, et al. Involvement of T cell Ig Mucin-3 (Tim3) in the negative regulation of inflammatory bowel disease. Clin Immunol 2010;134:169-177.

15. Nadkarni S, Mauri C, Ehrenstein MR. Anti-TNF-alpha therapy induces a distinct regulatory $\mathrm{T}$ cell population in patients with rheumatoid arthritis via TGF-beta. J Exp Med 2007;204:33-39.

16. Izcue A, Powrie F. Special regulatory T-cell review: regulatory $T$ cells and the intestinal tract--patrolling the frontier. Immunology 2008;123:6-10.

17. Holländer GA, Simpson SJ, Mizoguchi E, et al. Severe colitis in mice with aberrant thymic selection. Immunity 1995;3:27-38.

18. Mottet C, Uhlig HH, Powrie F. Cutting edge: cure of colitis by CD4+CD25+ regulatory T cells. J Immunol 2003;170:3939-3943.

19. Hori S, Nomura T, Sakaguchi S. Control of regulatory T cell development by the transcription factor Foxp3. Science 2003;299:10571061.

20. Fontenot JD, Gavin MA, Rudensky AY. Foxp3 programs the development and function of $\mathrm{CD} 4+\mathrm{CD} 25+$ regulatory $\mathrm{T}$ cells. Nat Immunol 2003;4:330-336.

21. Wehkamp J, Schmid M, Stange EF. Defensins and other antimi- crobial peptides in inflammatory bowel disease. Curr Opin Gastroenterol 2007;23:370-378.

22. Dhaliwal W, Bajaj-Elliott M, Kelly P. Intestinal defensin gene expression in human populations. Mol Immunol 2003;40:469-475.

23. Lehrer RI. Primate defensins. Nat Rev Microbiol 2004;2:727-738.

24. Siegmund B, Zeitz M. Innate and adaptive immunity in inflammatory bowel disease. World J Gastroenterol 2011;17:3178-3183.

25. Uhlig HH, McKenzie BS, Hue S, et al. Differential activity of IL-12 and IL-23 in mucosal and systemic innate immune pathology. Immunity 2006;25:309-318.

26. Hue S, Ahern P, Buonocore S, et al. Interleukin-23 drives innate and T cell-mediated intestinal inflammation. J Exp Med 2006;203:2473-2483.

27. Becker C, Dornhoff H, Neufert C, et al. Cutting edge: IL-23 crossregulates IL-12 production in T cell-dependent experimental colitis. J Immunol 2006;177:2760-2764.

28. IBD Working Group of the European Society for Paediatric Gastroenterology, Hepatology and Nutrition. Inflammatory bowel disease in children and adolescents: recommendations for diagnosis-the Porto criteria. J Pediatr Gastroenterol Nutr 2005;41:1-7.

29. Hyams JS, Ferry GD, Mandel FS, et al. Development and validation of a pediatric Crohn's disease activity index. J Pediatr Gastroenterol Nutr 1991;12:439-447.

30. Allen JE, Maizels RM. Th1-Th2: reliable paradigm or dangerous dogma? Immunol Today 1997;18:387-392.

31. Rook G. Th1- or Th2-cell commitment during infectious disease: an oversimplification? Trends Immunol 2001;22:481-482.

32. Anderson DE. TIM-3 as a therapeutic target in human inflammatory diseases. Expert Opin Ther Targets 2007;11:1005-1009.

33. Morimoto K, Hosomi S, Yamagami H, et al. Dysregulated upregulation of T-cell immunoglobulin and mucin domain-3 on mucosal T helper 1 cells in patients with Crohn's disease. Scand J Gastroenterol 2011;46:701-709.

34. Nakae S, likura M, Suto H, et al. TIM-1 and TIM-3 enhancement of Th2 cytokine production by mast cells. Blood 2007;110:25652568.

35. Cua DJ, Sherlock J, Chen Y, et al. Interleukin-23 rather than interleukin-12 is the critical cytokine for autoimmune inflammation of the brain. Nature 2003;421:744-748.

36. Anderson AC, Anderson DE, Bregoli L, et al. Promotion of tissue inflammation by the immune receptor Tim-3 expressed on innate immune cells. Science 2007;318:1141-1143.

37. Wang F, He W, Zhou H, et al. The Tim-3 ligand galectin-9 negatively regulates CD8+ alloreactive $\mathrm{T}$ cell and prolongs survival of skin graft. Cell Immunol 2007;250:68-74.

38. Sarin A, Conan-Cibotti M, Henkart PA. Cytotoxic effect of TNF and lymphotoxin on T lymphoblasts. J Immunol 1995;155:37163718.

39. Kelsen J, Agnholt J, Hoffmann HJ, Rømer JL, Hvas CL, Dahlerup JF. FoxP3(+)CD4(+)CD25(+) T cells with regulatory properties can be cultured from colonic mucosa of patients with Crohn's disease. Clin Exp Immunol 2005;141:549-557. 
40. Zelenay S, Lopes-Carvalho T, Caramalho I, Moraes-Fontes MF, Rebelo M, Demengeot J. Foxp3+ CD25- CD4 T cells constitute a reservoir of committed regulatory cells that regain CD25 expression upon homeostatic expansion. Proc Natl Acad Sci U S A 2005;102: 4091-4096.

41. Saruta M, Yu QT, Fleshner PR, et al. Characterization of FOXP3+CD4+ regulatory T cells in Crohn's disease. Clin Immunol 2007;125:281-290.

42. Makita S, Kanai T, Oshima S, et al. CD4+CD25bright T cells in human intestinal lamina propria as regulatory cells. J Immunol 2004;173:3119-3130.

43. Muratov V, Ulfgren AK, Engström M, et al. Decreased numbers of FoxP3-positive and TLR-2-positive cells in intestinal mucosa are associated with improvement in patients with active inflammatory bowel disease following selective leukocyte apheresis. J Gastroenterol 2008;43:277-282.

44. Li Z, Arijs I, De Hertogh G, et al. Reciprocal changes of Foxp3 ex- pression in blood and intestinal mucosa in IBD patients responding to infliximab. Inflamm Bowel Dis 2010;16:1299-1310.

45. Maul J, Loddenkemper C, Mundt P, et al. Peripheral and intestinal regulatory $\mathrm{CD} 4+\mathrm{CD} 25$ (high) $\mathrm{T}$ cells in inflammatory bowel disease. Gastroenterology 2005;128:1868-1878.

46. La Scaleia R, Morrone S, Stoppacciaro A, et al. Peripheral and intestinal CD4+ $\mathrm{T}$ cells with a regulatory phenotype in pediatric patients with inflammatory bowel disease. J Pediatr Gastroenterol Nutr 2010;51:563-572.

47. Doss M, White MR, Tecle T, Hartshorn KL. Human defensins and LL-37 in mucosal immunity. J Leukoc Biol 2010;87:79-92.

48. Bowdish DM, Davidson DJ, Hancock RE. Immunomodulatory properties of defensins and cathelicidins. Curr Top Microbiol Immunol 2006;306:27-66.

49. O'Neil DA, Porter EM, Elewaut D, et al. Expression and regulation of the human beta-defensins hBD-1 and hBD-2 in intestinal epithelium. J Immunol 1999;163:6718-6724. 